First published in:

\title{
Estimation of failure probabilities by local approximation of the limit state function
}

\author{
C. Proppe \\ Institut für Technische Mechanik, Universität Karlsruhe (TH), Kaiserstrasse 12, D-76131 Karlsruhe, Germany
}

Received 24 October 2005; received in revised form 19 January 2007; accepted 17 April 2007

Available online 19 June 2007

\begin{abstract}
For failure probability estimates of large structural systems, the numerical expensive evaluations of the limit state function have to be replaced by suitable approximations. Most of the methods proposed in the literature so far construct global approximations of the failure hypersurface. Rather than concentrating on the construction of the failure hypersurface, an adaptive local approximation scheme for the limit state function that is based on the moving least squares method is proposed in this study. It integrates well with existing importance sampling schemes and yields both efficient and robust estimates of the failure probability.
\end{abstract}

(C) 2008 Elsevier Ltd. All rights reserved.

Keywords: Response surface method; Moving least squares; Failure probability; Limit state function; Importance sampling

\section{Introduction}

The computation of failure probabilities by level III methods, such as Monte Carlo simulation (MCS) or Numerical Integration, requires a frequent evaluation of the limit state function, which is a numerically expensive operation for large structural models. In order to reduce the computational effort, a response surface method (RSM) is often employed, where an analytical expression is fitted by interpolation or regression to some evaluations of the limit state function. By means of Design of Experiments [1-4], the necessary evaluation points of the limit state function are obtained.

This approach can be traced back to Box and Wilson [5]. In context with reliability problems, first applications can be found in [6], where Faravelli (see also [7]) fits a response surface including the most influential random variables by means of a central composite design, while the other random variables are represented by their mean values. The quality of the approximation is assessed by statistical tests. In subsequent papers, e.g. [8-12], the aspect that the response surface includes only a part of the random variables has not been further pursued and the response surface methods proposed in these papers are purely interpolation or regression methods.

E-mail address: proppe@itm.uka.de 
In [8], the main interest is an approximation of the limit state function in the vicinity of the point of most probable failure (MPP), i.e., the point on the failure hypersurface with maximum likelihood. Bucher and Bourgund [8] propose an interpolation with an incomplete second order polynomial (neglecting the crossterms) and an update of the response surface that is based on evaluation points centered around the point that lies on the failure hypersurface and on a straight line that links the mean values of the random variables with the approximate MPP. The main advantage of this algorithm is that the computational effort increases with $O(n)$, where $n$ is the number of random variables. Contrary to this interpolation polynomial, Wong [13] applies an incomplete second order polynomial with cross-terms, but where the square terms are omitted. Rajashekhar and Ellingwood [9] pointed out, that the results depend on the distance between the evaluation points and on the shape of the limit state function and that a criterion for the approximation quality of the response surface is missing. Therefore, they propose an adaptive selection of experimental designs, introduce the distance between the center point of the experiments and the approximate MPP as quality criterion and elaborate a criterion that helps to decide whether a cross term should be neglected in the interpolation polynomial or not. Moreover, they investigate experiments at distribution extremes, but conclude that there are no advantages with respect to computational effort and accuracy.

Kim and $\mathrm{Na}[10]$ try to iterate with linear response surfaces and project the evaluation points on the approximate failure hypersurface. Zheng and Das [11] improve this approach by adding square terms to the interpolation polynomial and a quality check. They introduce the idea to reuse the information at formerly evaluated points. Later, Gayton et al. [12] also consider the reuse of formerly evaluated points and elaborate an algorithm for iterative MPP estimates based on resampling of experiments. However, due to the use of a complete second order response surface, the computational effort is of $O\left(n^{2}\right)$.

The above mentioned response surface methods differ in the choice of the interpolation polynomial and of the evaluation points and it has been shown [14] that the obtained results are highly dependent on these choices and on the shape of the actual failure hypersurface. According to Hurtado and Alvarez [15], this is due to the rigid and non-adaptive structure of the models implemented by response surface methods. Moreover, it is noted that global approximations are often constructed for parameter spaces that ignore constraints imposed by the physical nature of the problem. Proposals to circumvent these problems encompass iterative updating of the response surface [9-12], introduction of hyperplane approximations [16-18] and of artificial neural networks [19-22]. Hyperplane approximation methods show a tendency to link importance sampling methods and response surfaces, but are limited by the necessity to find evaluation points that lie on the failure hypersurface and by gradient computations. Regarding neural networks, [15,22] report that radial basis functions perform better than backpropagation multilayer perceptrons, since the former can better represent local information. However, artificial neural networks need a properly chosen training set.

From the references cited above, it can be concluded that an approximation of the limit state function or the failure hypersurface must be flexible enough to provide highly accurate results close to the MPP and rather coarse approximations elsewhere. Therefore, the approximation procedure must be adaptive, and a criterion to assess the quality of the solution is mandatory. In order to deal with problems involving, even after a suitable reduction, a considerable large amount of random variables, it is necessary to reuse the information at previously evaluated points and to predict only what must be predicted: the sign of the limit state function for the samplings provided by an importance sampling scheme.

The moving least squares method (MLS) $[23,24]$ seems to be an ideal candidate for a flexible approximation scheme. By selection of a few parameters, cf. Section 3, it can provide accurate as well as coarse approximations, depending on the region of interest. Moreover, it can be easily combined with importance sampling schemes.

In this study, a robust and efficient local approximation scheme of the limit state function that is based on the MLS for the estimation of failure probabilities is proposed. The major advantages of the proposed local approximation are that

(1) the limit state function is evaluated close to the region of most likely failure only;

(2) it is not necessary to compute zeros of the limit state function;

(3) all evaluation points are reused;

(4) there is an interaction between importance sampling schemes and the limit state approximation; 
(5) the quality of the approximation can be assessed and a convergence criterion can be established;

(6) the procedure is easy to implement.

The algorithm consists of four basic parts (cf. Fig. 1):

(1) an initial optimization algorithm that directs the simulation to the region(s) of most likely failure;

(2) a local approximation algorithm for the limit state function that is based on the data points obtained in step 1;

(3) an importance sampling algorithm that makes use of the results obtained in step 1 and the local interpolation of the limit state function of step 2;

(4) an adaptation procedure in order to improve the importance sampling scheme and/or the quality of the interpolation result.

While the optimization schemes, the moving least squares approximation and the importance sampling schemes are well established in their respective areas of application, they are linked in this paper by an adaptation procedure, for which a quality measure based on a ranking is provided. The following sections introduce and discuss suitable implementations for the parts of the algorithm. After that, several examples are given in order to illustrate the efficiency of the proposed method.

\section{Optimization algorithm}

The problem addressed in this paper is the approximation of the probability of failure of a structure, which may be defined as

$$
P_{\mathrm{F}}=\int_{\mathbb{F}} p(\boldsymbol{\theta}) \mathrm{d} \boldsymbol{\theta}
$$

where $p(\boldsymbol{\theta})$ is the joint probability density function of the vector of random parameters $\boldsymbol{\theta}, \mathbb{F}=\{\boldsymbol{\theta} \mid g(\boldsymbol{\theta}) \leqslant 0\}$ denotes the failure domain and $g(\theta)$ the limit state function. In engineering practice, the limit state function

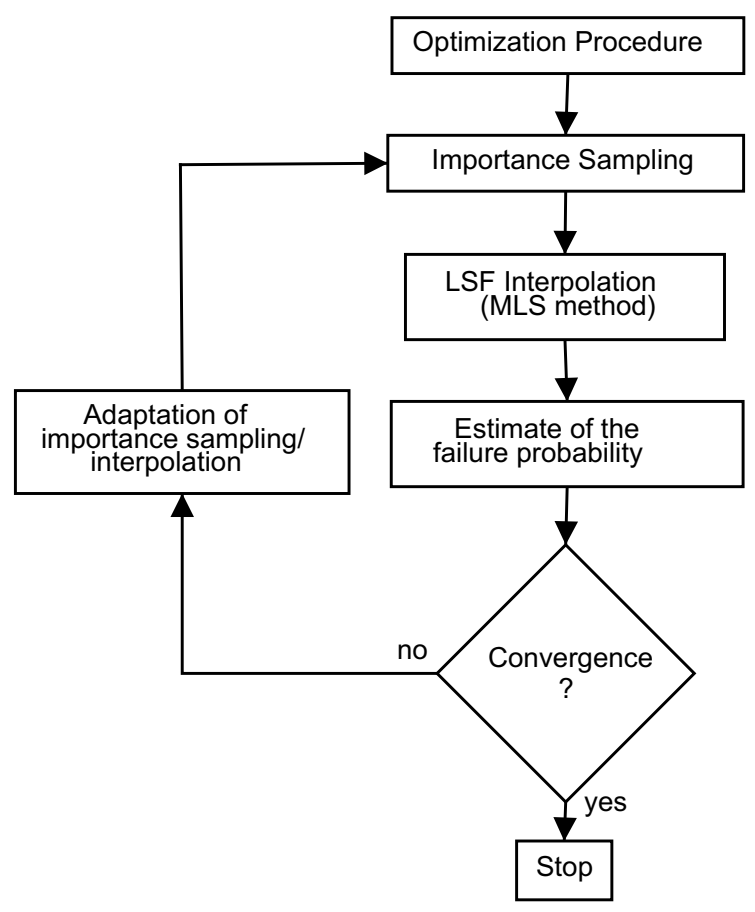

Fig. 1. Flow chart of the algorithm. 
may be given in implicit form only, e.g. as a result of a stability analysis, or constructed as the union of several failure domains, each representing a structural failure mode. For large finite element systems, the evaluation of the limit state function may be very time consuming and should be reduced to a minimum.

The probability of failure may be estimated from MCS. Usually, the major contributions to the integral in Eq. (1) stem from regions around the MPPs. Efficient simulation techniques try to concentrate the sample points in these regions. The computation of the MPPs is an optimization problem, that can be solved by non-linear programming techniques or evolutionary methods.

\subsection{Non-linear programming}

The MPP can be obtained from an optimization problem with an equality constraint by eventually transforming the random variables and the limit state function to standard normal space. Several algorithms can be applied for solving this non-linear constraint programming problem, see e.g. [25] for details.

\subsection{Metropolis algorithm}

Starting from a sample point that falls in the failure domain, the Metropolis algorithm [26] produces sample points that cover the region of most likely failure. Let $\tilde{p}(\xi \mid \boldsymbol{\theta})$ be a given probability density function (p.d.f.) that is symmetric with respect to its arguments. Starting from a sample point $\boldsymbol{\theta}_{i}$ lying in the failure domain, the successor $\boldsymbol{\theta}_{i+1}$ is obtained in the following way: first, a sample point $\boldsymbol{\xi}$ is generated from $\tilde{p}\left(\boldsymbol{\xi} \mid \boldsymbol{\theta}_{i}\right)$ and the ratio $\rho=I_{g<0}(\xi) p(\xi) / p\left(\boldsymbol{\theta}_{i}\right)$ is computed. The indicator function $I_{g<0}(\boldsymbol{\theta})$ is 1 in the failure domain and zero elsewhere. If $\rho$ is greater than or equal to one, then $\boldsymbol{\theta}_{i+1}=\boldsymbol{\xi}$. However, if $\rho$ is smaller than one, $\boldsymbol{\theta}_{i+1}=\boldsymbol{\xi}$ with probability $\rho$ and $\boldsymbol{\theta}_{i+1}=\boldsymbol{\theta}_{i}$ with probability $1-\rho$.

It can be shown [27] that the algorithm generates a first-order Markov chain. Moreover, the asymptotic distribution of $\boldsymbol{\theta}$ is given by the optimal sampling density $I_{g<0}(\boldsymbol{\theta}) p(\boldsymbol{\theta}) / P_{\mathrm{F}}$ [28]. Unfortunately, the asymptotic result is useless in the context of this study, as the convergence speed towards the optimal sampling density is decisive. However, the convergence speed can be influenced by several parameters, which are discussed in the following.

Contrary to what has been stated in [28], experiences indicated that the type of the p.d.f. $\tilde{p}(\xi \mid \boldsymbol{\theta})$ has a great importance for the robustness of the simulation scheme. Especially, it has been found that bell-shaped p.d.f.s yield a more robust scheme, while the results obtained with an $n$-dimensional uniform p.d.f. were very sensitive with respect to the side lengths of the hyper cube for which $\tilde{p}(\xi \mid \boldsymbol{\theta})>0$. In general, assuming for $\tilde{p}(\boldsymbol{\xi} \mid \boldsymbol{\theta})>0$ a product of $n$ unidimensional Gaussian p.d.f.s with mean $\theta_{i}, i=1, \ldots, n$, and standard deviation $\sigma_{i}$, $i=1, \ldots, n$, is a good choice, as it allows for rather short steps (with a higher probability) but also larger steps. The parameters $\sigma_{i}$ affect the distance the next sample point can depart from the actual one. Choosing $\sigma_{i}$ too large will yield a large spread of the generated data points, with many sample points falling in unimportant regions. On the other hand, if $\sigma_{i}$ is too small, the convergence will be very slow and chances are high that after a certain number of generated sample points the important regions are not covered by the sample points.

To speed up the algorithm, it is possible to introduce a relaxation parameter $\gamma>0$, such that the next sample point is accepted with probability $\min (0, \gamma \rho)$ and rejected with probability $\max (0,1-\gamma \rho)$. Obviously, $\gamma>1$ corresponds to a more progressive and $0<\gamma<1$ to a more conservative algorithm.

As mentioned above, the proper choice of the parameters $\gamma$ and $\sigma_{i}$ is crucial for the convergence speed of the algorithm and may vary from problem to problem. Thus, it might be important to adapt the parameters in course of the simulation procedure. For sample points $\xi$ that fall in the failure domain, the parameter $\gamma$ may be modified as follows: if $\gamma_{\text {old }} \rho>1$, then $\gamma_{\text {new }}=1+\frac{\gamma_{\text {old }}-1}{\epsilon_{\gamma}}$, else $\gamma_{\text {new }}=1+\epsilon_{\gamma}\left(\gamma_{\text {old }}-1\right)$, where $\epsilon_{\gamma}$ is slightly greater than 1, i.e. between 1 and 1.5. In the same way, the standard deviation $\sigma_{i}$ may be adapted: if $\rho>1$

$$
\sigma_{\text {inew }}=\frac{\frac{\sigma_{\text {iold }}}{\epsilon_{\mathrm{abs}}}+\frac{\left|\theta_{i}-\xi_{i}\right|}{\epsilon_{\mathrm{rel}}}}{\frac{1}{\epsilon_{\mathrm{abs}}}+\frac{1}{\epsilon_{\mathrm{rel}}}},
$$

else

$$
\sigma_{\text {inew }}=\frac{\sigma_{\text {iold }} \epsilon_{\mathrm{abs}}+\left|\theta_{i}-\xi_{i}\right| \epsilon_{\mathrm{rel}}}{\epsilon_{\mathrm{abs}}}
$$



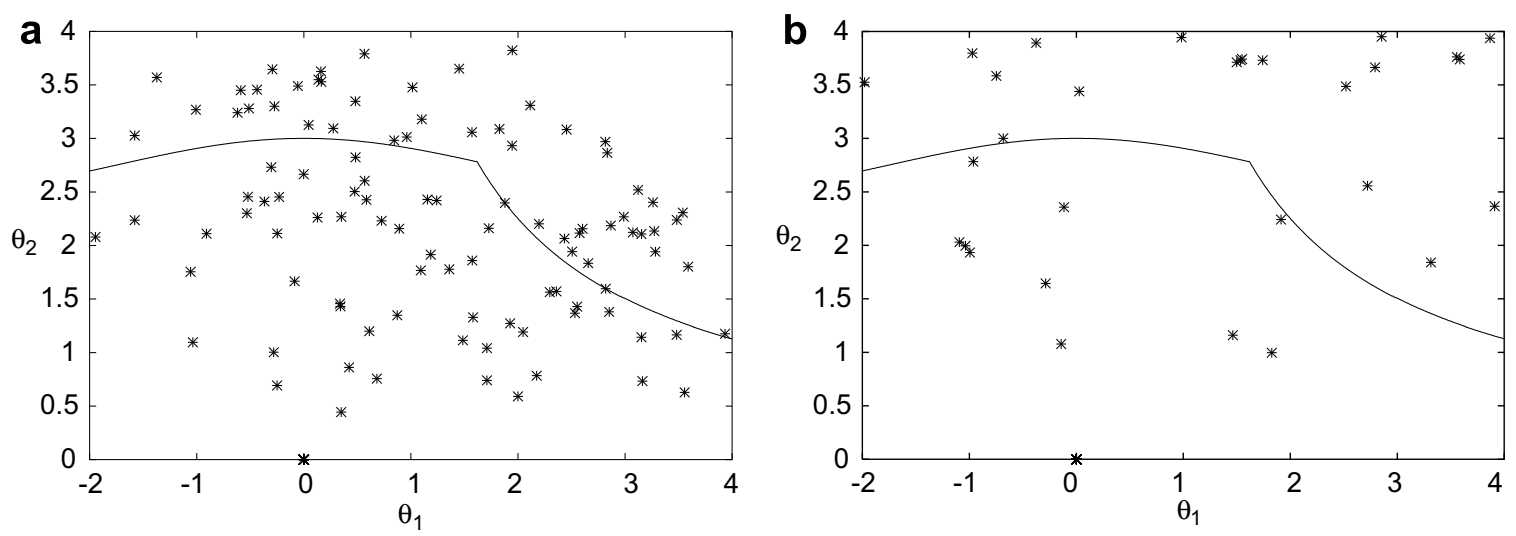

Fig. 2. One hundred and fifty samples generated by the metropolis algorithm with (a) $\epsilon_{\mathrm{abs}}=1.1, \epsilon_{\mathrm{rel}}=0.75, \sigma_{1}=\sigma_{2}=1$ and (b) $\epsilon_{\mathrm{abs}}=1$, $\epsilon_{\text {rel }}=0.95, \sigma_{1}=\sigma_{2}=0.5$. Solid lines: failure hypersurface $g\left(\theta_{1}, \theta_{2}\right)=0$.

where usually $\epsilon_{\mathrm{abs}}$ is greater than 1 (between 1 and 1.5) and $\epsilon_{\mathrm{rel}}$ smaller than 1 (between 0.5 and 1). This formulation accounts for an absolute as well as a relative change of $\sigma_{i}$ with respect to the generated sample points. In order to not produce excessively large or small standard deviations, formula (2) and (3) should not be directly repeated more than twice.

Also the initial sample point $\boldsymbol{\theta}_{0}$ may have a considerable influence on the convergence speed. It is in general chosen according to the engineer's best knowledge and is not subject to modifications.

Example. Consider the limit state function [28]

$$
g\left(\theta_{1}, \theta_{2}\right)=\min \left(c-1-\theta_{2}+\exp \left(-\frac{\theta_{1}^{2}}{10}\right)+\left(\frac{\theta_{1}}{5}\right)^{4}, \frac{c^{2}}{2}-\theta_{1} \theta_{2}\right),
$$

where $c$ is a constant and $\theta_{1}, \theta_{2}$ are standard normally distributed random variables. For $c=3$, the system has two MPPs, situated at $(0,3)$ and $(3 / \sqrt{2}, 3 / \sqrt{2})$. As Fig. 2 indicates, depending on the parameters of the algorithm (the starting points as well as $\gamma$ were the same for both cases), few sample points may be sufficient to cover the region of most probable failure.

\section{Local approximation based on MLS}

The MLS approximation scheme $[23,24]$ has been frequently employed in conjunction with meshless methods. It has a reasonably high accuracy and can be easily extended to problems of arbitrary large dimension. Consider a neighborhood $\Omega_{\bar{\theta}}$ of the sample point $\overline{\boldsymbol{\theta}}$, for which the MLS approximation $\bar{g}(\boldsymbol{\theta})$ of the limit state function should be calculated. For $\theta \in \Omega_{\bar{\theta}}$, the MLS approximant is defined by $\bar{g}(\boldsymbol{\theta})=\boldsymbol{p}^{\mathrm{T}}(\boldsymbol{\theta}) \boldsymbol{a}(\overline{\boldsymbol{\theta}})$, where $\boldsymbol{p}(\boldsymbol{\theta})$ is a complete monomial basis of order $m$ and $\boldsymbol{a}(\overline{\boldsymbol{\theta}})$ contains the $m$ interpolation coefficients, which are determined from the $n$ given data points $\boldsymbol{\theta}_{\boldsymbol{i}}, i=1, \ldots, n$, by minimizing the weighted discrete $L_{2}$ norm

$$
[\boldsymbol{P a}(\overline{\boldsymbol{\theta}})-\hat{\boldsymbol{g}}]^{\mathrm{T}} \boldsymbol{W}(\overline{\boldsymbol{\theta}})[\boldsymbol{P a}(\overline{\boldsymbol{\theta}})-\hat{\boldsymbol{g}}],
$$

where $\boldsymbol{P}$ is the $n \times m$ dimensional matrix

$$
\left[\begin{array}{c}
\boldsymbol{p}^{\mathrm{T}}\left(\boldsymbol{\theta}_{1}\right) \\
\boldsymbol{p}^{\mathrm{T}}\left(\boldsymbol{\theta}_{2}\right) \\
\vdots \\
\boldsymbol{p}^{\mathrm{T}}\left(\boldsymbol{\theta}_{n}\right)
\end{array}\right]
$$


$\boldsymbol{W}(\overline{\boldsymbol{\theta}})$ is the $n \times n$ dimensional diagonal matrix containing the values of the weight functions $w_{i i}=w\left(\boldsymbol{\theta}_{i}-\overline{\boldsymbol{\theta}}\right)>0$ and the $n$ dimensional vector $\hat{\boldsymbol{g}}$ contains the values of the limit state function at the sample points $\boldsymbol{\theta}_{\boldsymbol{i}}, i=1, \ldots, n$. The minimization procedure leads to the following linear system for the interpolation coefficients

$$
\boldsymbol{A}(\overline{\boldsymbol{\theta}}) \boldsymbol{a}(\overline{\boldsymbol{\theta}})=\boldsymbol{B}(\overline{\boldsymbol{\theta}}) \hat{\boldsymbol{g}}
$$

with

$$
\boldsymbol{A}(\overline{\boldsymbol{\theta}})=\boldsymbol{P}^{\mathrm{T}} \boldsymbol{W}(\overline{\boldsymbol{\theta}}) \boldsymbol{P} \text { and } \boldsymbol{B}(\overline{\boldsymbol{\theta}})=\boldsymbol{P}^{\mathrm{T}} \boldsymbol{W}(\overline{\boldsymbol{\theta}}),
$$

that has a unique solution if and only if matrix $\boldsymbol{A}(\boldsymbol{\theta})$ is regular, i.e. of rank $m$. Thus, a necessary condition for a unique solution is that at least $m$ weight functions are non-zero.

The weight functions are positive with compact supports. A suitable choice are Gaussian weight functions

$$
w(d)= \begin{cases}\frac{\exp \left[-(d / \sigma)^{2}\right]-\exp \left[-(r / \sigma)^{2}\right]}{1-\exp \left[-(r / \sigma)^{2}\right]}, & 0 \leqslant d \leqslant r \\ 0, & d \geqslant r\end{cases}
$$

or spline weight functions

$$
w(d)=\left\{\begin{array}{ll}
1-6\left(\frac{d}{r}\right)^{2}+8\left(\frac{d}{r}\right)^{3}-3\left(\frac{d}{r}\right)^{4}, & 0 \leqslant d \leqslant r \\
0, & d \geqslant r
\end{array},\right.
$$

that depend only on the Euclidean norm $d=\left\|\boldsymbol{\theta}_{i}-\overline{\boldsymbol{\theta}}\right\|$. In this study, spline weight functions are used exclusively, as the Gaussian weight functions contain an additional parameter $\sigma$, which is difficult to determine unless it is not directly related to $r$.

The size of the support $r$ determines the local character of the approximation. If $r$ is too small, then the regularity of $\boldsymbol{A}(\overline{\boldsymbol{\theta}})$ may not be assured. On the other hand, if $r$ is too large, the local character of the interpolation scheme is violated. The quantity $r$ may vary according to whether or not $\overline{\boldsymbol{\theta}}$ is close to the failure hypersurface. As long as there is a common scale factor, $r$ can be used to introduce a quality measure of the approximation with respect to a given sample point. From the fact that the local interpolation should be well defined for all sample points, one can obtain a minimal value for the scale factor. By comparing the scale factors of different sample points, the accuracy of the estimates of the probability of failure with respect to the underlying approximation can be assessed.

\section{Importance sampling}

The integral appearing in Eq. (1) can be written as

$$
P_{\mathrm{F}}=\int_{\mathbb{E}} \frac{p(\boldsymbol{\theta})}{\tilde{p}(\boldsymbol{\theta})} \tilde{p}(\boldsymbol{\theta}) \mathrm{d} \boldsymbol{\theta}
$$

where the weighting density function $\tilde{p}(\boldsymbol{\theta})$ has been introduced. The purpose of the weighting function is to reduce the variance of the estimator

$$
\widehat{P}_{\mathrm{F}}=\frac{1}{N} \sum_{i=1}^{N} I_{g\left(\boldsymbol{\theta}_{i}\right) \leqslant 0} p\left(\boldsymbol{\theta}_{i}\right),
$$

from simulated results. Theoretically, the variance can be reduced to zero, but this would require a priori knowledge of the p.d.f. $p(\theta)$ in the failure domain.

\subsection{Importance sampling using MPPs}

A good choice for the weighting function are multi-normal p.d.f.s centered around the MPPs. The variances should not be too small; they can be set according to engineering judgement or by means of adaptation strategies [29]. 


\subsection{Kernel sampling density algorithm}

Starting from the initial sample points obtained as described in Section 2, a kernel sampling density is constructed as in [30] with adaptive bandwidth parameters as recently introduced in [28]:

$$
k(\boldsymbol{\theta})=\frac{1}{M} \sum_{i=1}^{M} \frac{1}{\left(w \lambda_{i}\right)^{n}} p_{N}\left(\frac{\boldsymbol{\theta}-\boldsymbol{\theta}_{i}}{w \lambda_{i}}\right),
$$

where $p_{N}(\boldsymbol{\theta})$ is the Gaussian p.d.f.

$$
p_{N}(\boldsymbol{\theta})=\frac{1}{\sqrt{(2 \pi)^{n} \operatorname{det}(\boldsymbol{\mu})}} \exp \left(-\frac{1}{2} \boldsymbol{\theta}^{\mathrm{T}} \boldsymbol{\mu}^{-1} \boldsymbol{\theta}\right),
$$

with the covariance matrix $\mu$ of the sample points $\theta_{i}, i=1, \ldots, M$. Thus, the sampling density is a sum of Gaussian densities constructed in each of the sample points $\theta_{i}, i=1, \ldots, M$, whose domain of influence is controlled by the factor $w \lambda_{i}$. With a suitable choice of $\lambda_{i}$, a larger zone of influence can be generated for regions of lower probability density, while $w$ can be adapted to reduce the variance of the estimator for the failure probability. According to [28], an optimal value for $\lambda_{i}$ is

$$
\lambda_{i}=\left[\left(\prod_{j=1}^{M} p\left(\boldsymbol{\theta}_{j}\right)\right)^{1 / M} / p\left(\boldsymbol{\theta}_{i}\right)\right]^{\alpha},
$$
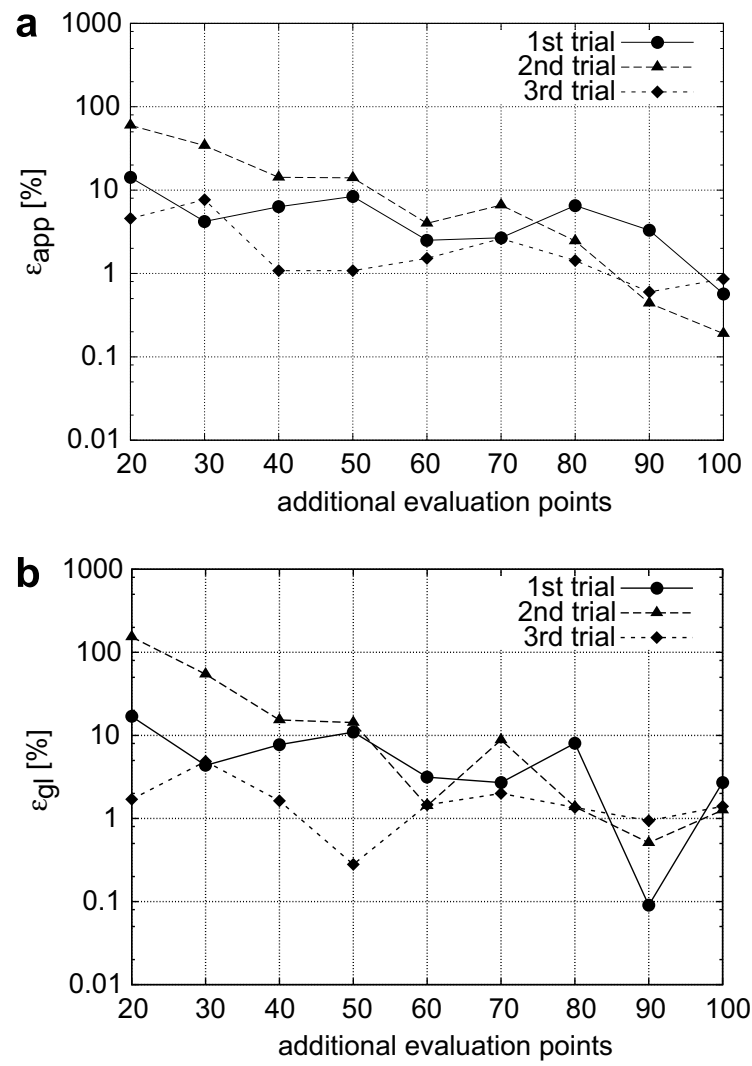

Fig. 3. Relative errors $\epsilon_{\mathrm{app}}$ and $\epsilon_{\mathrm{gl}}$, as a function of the number of evaluation points. (a) $\epsilon_{\mathrm{app}}$ : relative error of the local approximation; (b) $\epsilon_{\mathrm{gl}}$ : relative error of the proposed method (importance sampling and local approximation). 
with the sensitivity parameter $\alpha, 0 \leqslant \alpha \leqslant 1$, and $w$ can be obtained from minimization of

$$
\sum_{i=1}^{M} \frac{p\left(\boldsymbol{\theta}_{i}\right)}{k_{-i}\left(\boldsymbol{\theta}_{i}\right)}
$$

where $k_{-i}\left(\boldsymbol{\theta}_{i}\right)$ is the kernel sampling density without the normal p.d.f. constructed in $\boldsymbol{\theta}_{i}$.
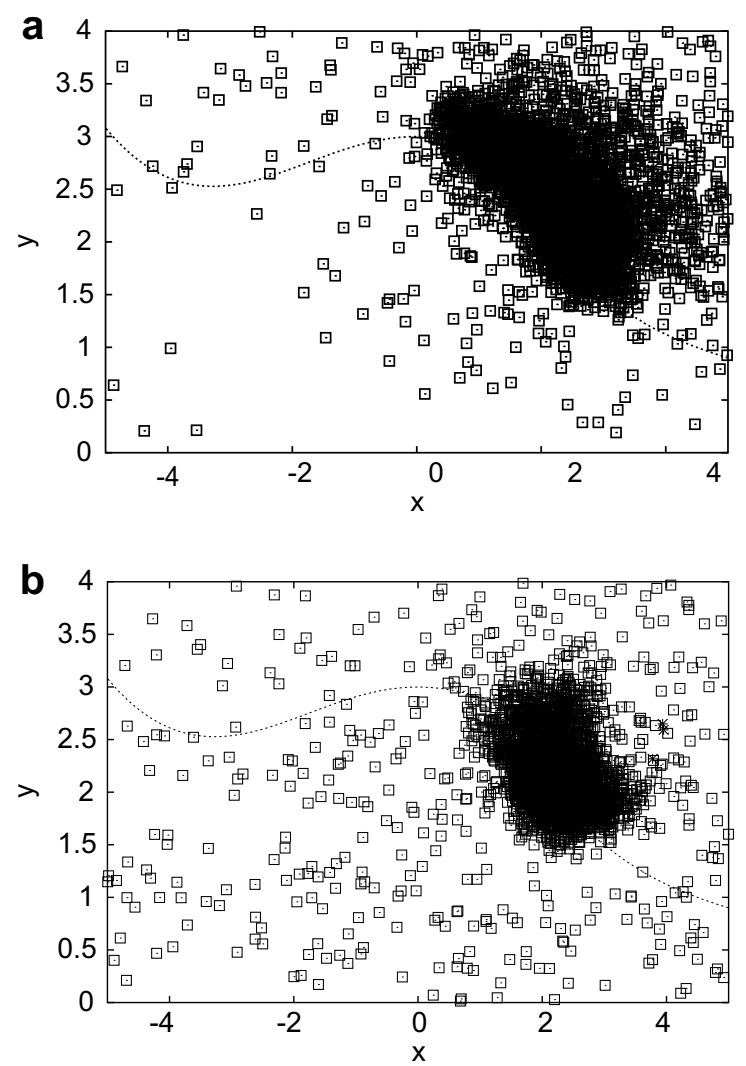

Fig. 4. Initial sampling (5000 samples) after completion of the optimization procedure (Metropolis algorithm). (a) First trial; (b) second trial.

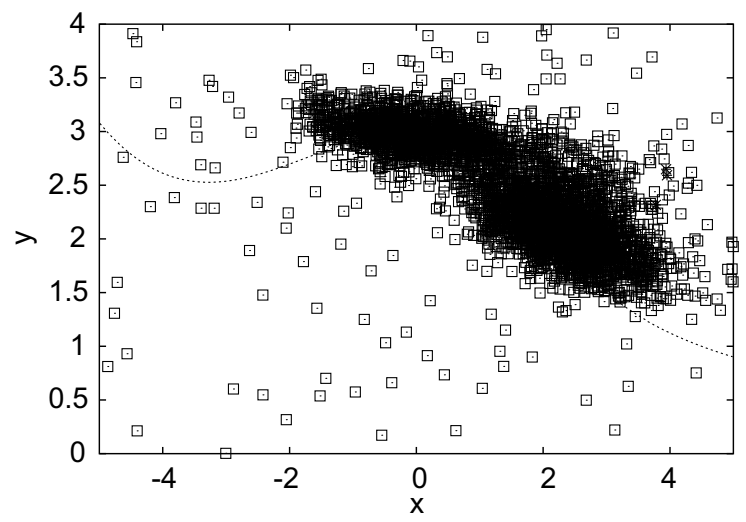

Fig. 5. Second trial: spatial distribution of the 5000 sample points after completion of the optimization procedure and five additional adaptation steps. 


\section{Adaptivity}

The adaptation procedure proposed here is based on an evolutionary strategy, where the created sample points are ranked according to their quality with respect to the estimation of the failure probability. An importance sampling point $\boldsymbol{\theta}_{i}$, for which the value of the limit state function can be predicted with relatively high uncertainty only is characterized by the following three properties:

- the sample point is close to the failure hypersurface, i.e. $\left|\bar{g}\left(\boldsymbol{\theta}_{i}\right)\right|$ is small;

- the probability $p\left(\boldsymbol{\theta}_{i}\right)$ of the sample point is relatively large;

- the minimal support size $r_{i}$ for that sample is relatively large.

From these properties, one can define a ranking of the generated sample points with respect to the uncertainty they introduce when the failure probability is estimated. For this, the sample points are ranked with respect to $\left|\bar{g}\left(\boldsymbol{\theta}_{i}\right)\right|, p\left(\boldsymbol{\theta}_{i}\right)$ and $r_{i}$, respectively and the results of the rankings are multiplied with a weight, added and ranked once again. As numerical experiments indicate, the choice of the weight is not crucial for the convergence velocity of the algorithm, if the first property has at least as much weight as the second or third criterion.

For the sample points which introduce the highest amount of uncertainty, the exact value of the limit state function is calculated. These sample points can be added to the base values of the interpolation algorithm.

Moreover, the sample points in the failure domain, but closest to the failure hypersurface with highest probability are used in the next iteration step as base sample points for the importance sampling scheme.
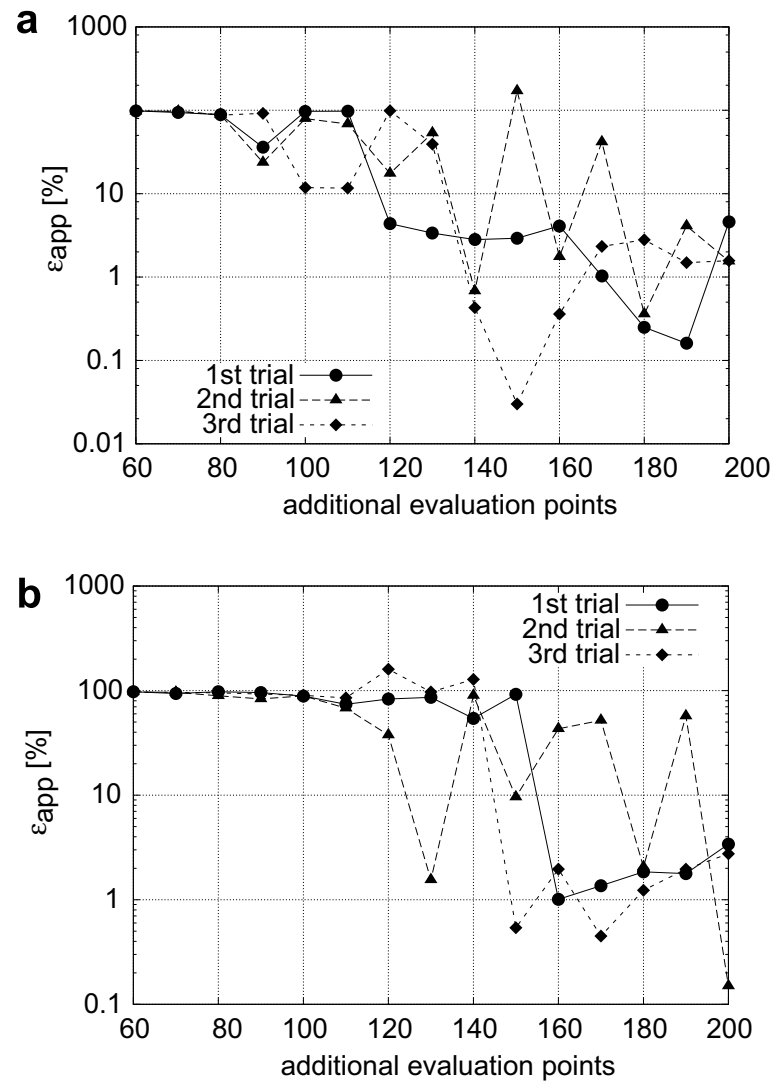

Fig. 6. Relative errors $\epsilon_{\mathrm{app}}$ of the local approximation, as a function of the number of evaluation points. (a) 5000 sample points; (b) 20,000 sample points. 
In this way, an interaction between the MLS approximation of the limit state function and the importance sampling scheme is obtained.

\section{Examples}

The first example deals with the limit state function already introduced in Eq. (4). The probability of failure for this example, $2.53 \times 10^{-3}$, has been obtained by direct MCS using sufficiently large sample sizes. In order to evidence the approximation quality of the MLS method, importance sampling with standard normal densities at the MPPs is considered. The initial evaluation points for the MLS approximation were arbitrarily generated from the importance sampling density, while in each step, 10 more evaluation points were added using the adaptation procedure described above. The approximations were started from 5 initial evaluation points, that were determined by simulation. The estimates were obtained from 20,000 sample points. The weights for the three criteria stated in Section 5 were $0.6,0.3$ and 0.1, respectively and 10 points were used for the MLS regression. The following relative errors have been evaluated:

$$
\epsilon_{\mathrm{app}}=\frac{\left|\hat{p}_{\mathrm{F}}-\hat{\bar{p}}_{\mathrm{F}}\right|}{\hat{\bar{p}}_{\mathrm{F}}}
$$

and

$$
\epsilon_{\mathrm{gl}}=\frac{\left|\hat{\bar{p}}_{\mathrm{F}}-p_{\mathrm{F}}\right|}{p_{\mathrm{F}}}
$$
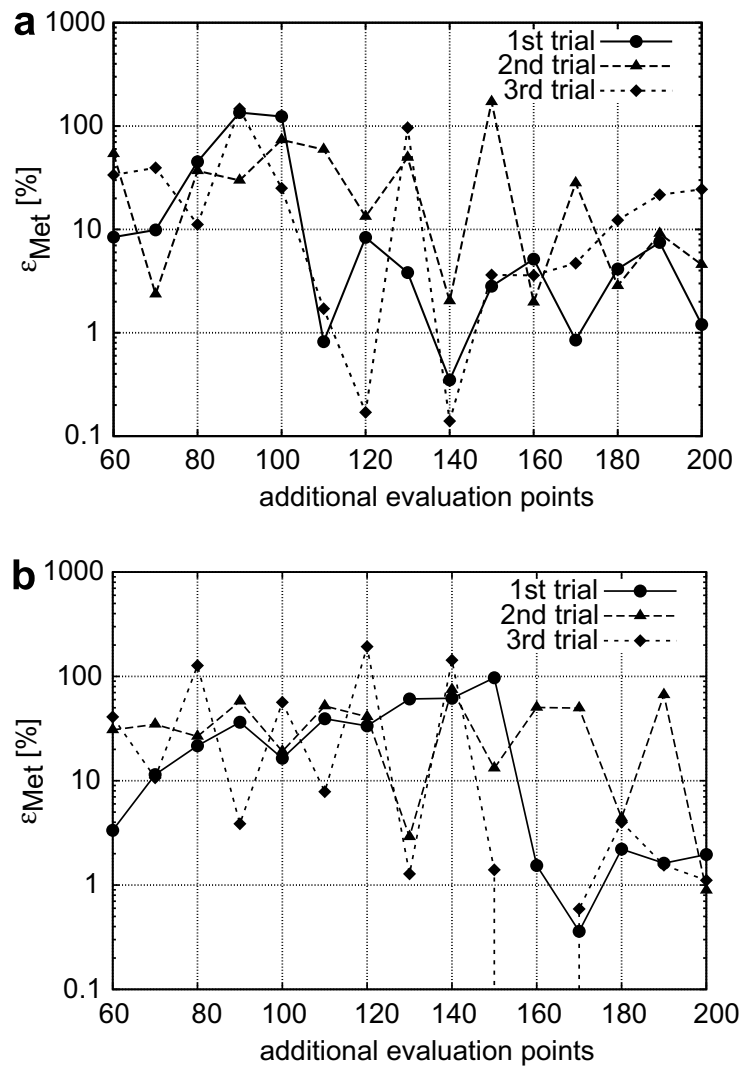

Fig. 7. Relative errors $\epsilon_{\text {Met }}$ of the importance sampling scheme, as a function of the number of evaluation points. (a) 5000 sample points; (b) 20,000 sample points. 
with $p_{\mathrm{F}}=2.53 \times 10^{-3}$ and the estimated probability of failure obtained by evaluating the limit state function $\left(\hat{p}_{\mathrm{F}}\right)$ and by approximating the limit state function $\left(\hat{\bar{p}}_{\mathrm{F}}\right)$ using the MLS scheme. $\epsilon_{\mathrm{gl}}$ measures the overall error between the exact solution and the proposed procedure (importance sampling and local approximation), while $\epsilon_{\text {app }}$ takes only the effect due to local approximation into account. From Fig. 3, it can be seen that the error decreases rapidly with growing number of additional evaluation points. For this two-dimensional example, one can conclude that the algorithm yields a reasonable small approximation error after only 50 additional limit state function evaluations.

Next, coupling of the local approximation with the Metropolis algorithm has been investigated. The starting points and the parameters of the Metropolis algorithm were chosen in such a way that for the first trial, both MPPs were detected, while for the second trial, only one MPP was detected at the beginning of the sampling, cf. Fig. 4. From Fig. 5, taken after 5 adaptation steps, it can be seen that the proposed adaptation procedure is capable of detecting the second MPP and modifying the sampling density such that the important region is uniformly covered by the sample points.

In order to judge the predictions obtained with the Metropolis algorithm, an additional relative error,

$$
\epsilon_{\mathrm{Met}}=\frac{\left|\hat{p}_{\mathrm{F}}-p_{\mathrm{F}}\right|}{p_{\mathrm{F}}}
$$

is introduced. Figs. 6-8 display results for $\epsilon_{\mathrm{app}}, \epsilon_{\mathrm{Met}}$ and $\epsilon_{\mathrm{gl}}$ obtained with 5000 and 200,000 sample points, respectively. Although the abscissa for $\epsilon_{\text {Met }}$ in Fig. 7 is meaningless (as limit state function evaluations are carried out for every sample point), it has been kept for comparison purposes with Figs. 6 and 8 .

Figs. 7 and 8 indicate that the approximation quality for the first and second trial is only slightly different, despite the fact that importance sampling for the second trial starts from one MPP only. Between 140 and 170
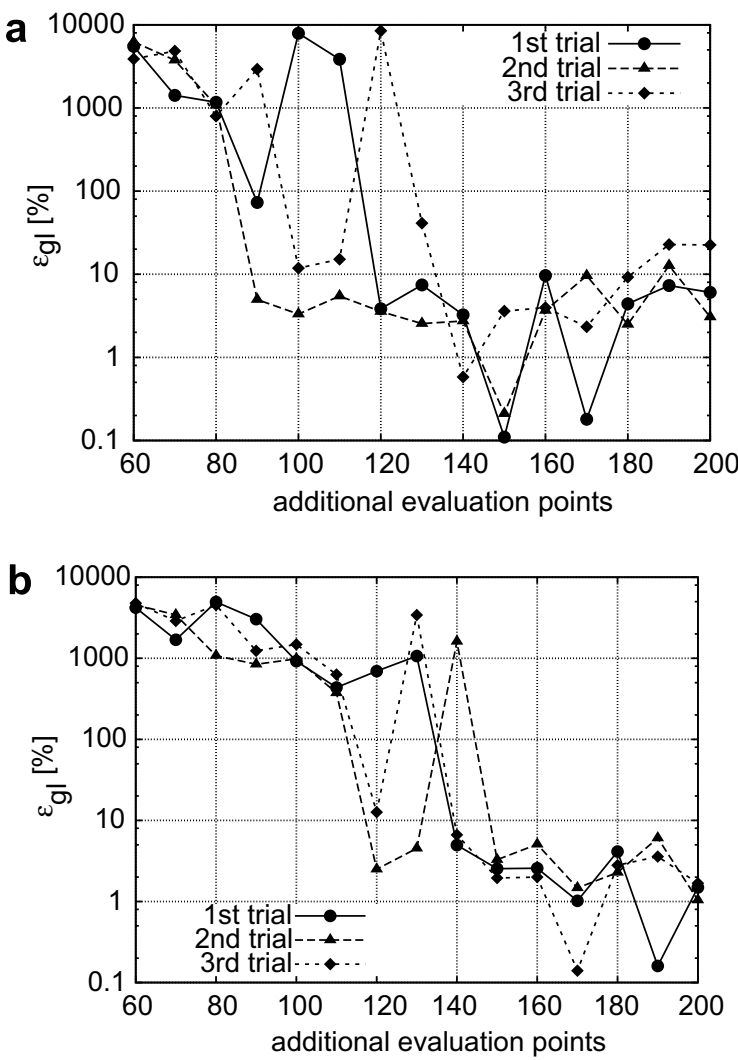

Fig. 8. Relative errors $\epsilon_{\mathrm{gl}}$ of the proposed procedure (importance sampling and local approximation), as a function of the number of evaluation points. (a) 5000 sample points; (b) 20,000 sample points. 
additional evaluation points, this difference is more pronounced for $\epsilon_{\mathrm{Met}}$ and is reduced for $\epsilon_{\mathrm{gl}}$ by a beneficial superposition of local approximation error and sampling error.

Fig. 6 indicates that the error due to local approximation of the limit state function drops sharply after 150 additional evaluation points. At the same time, also the Metropolis algorithm reaches a better approximation quality (cf. Fig. 7). After 150 additional evaluation points, the approximation error remains low, i.e. less than 10\%. As Fig. 9 indicates, convergence of the estimates for the failure probability can be detected based on a
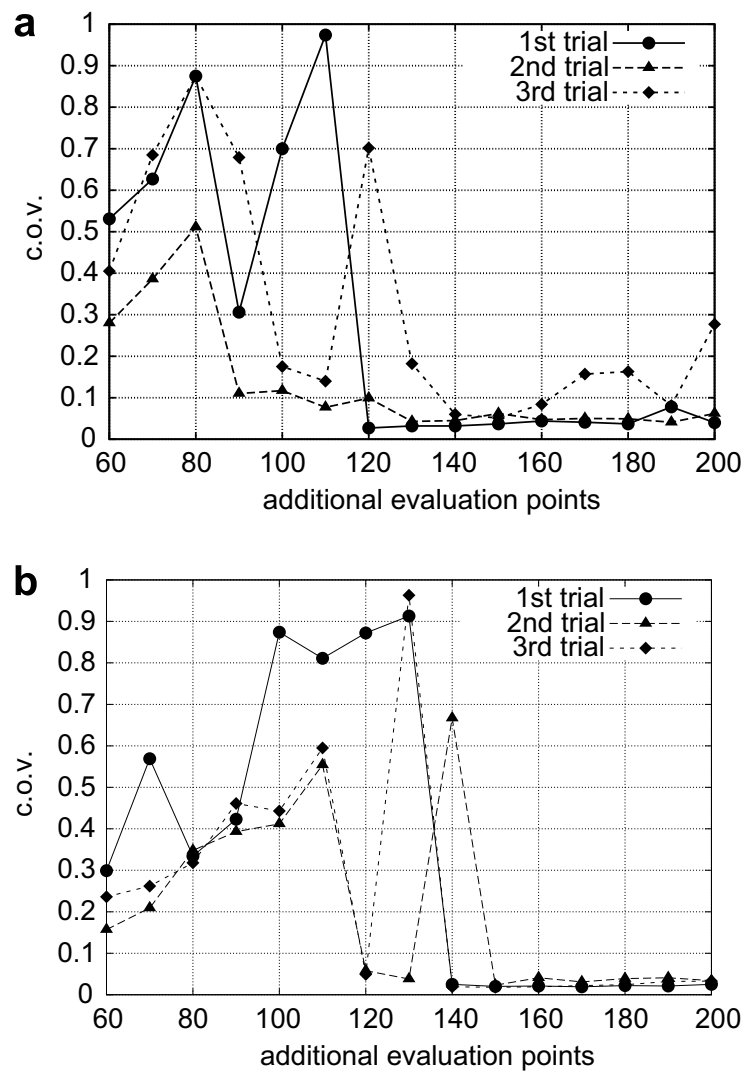

Fig. 9. Coefficients of variation for the probability of failure obtained with the proposed procedure (importance sampling and local approximation), as a function of the number of evaluation points. (a) 5000 sample points; (b) 20,000 sample points.

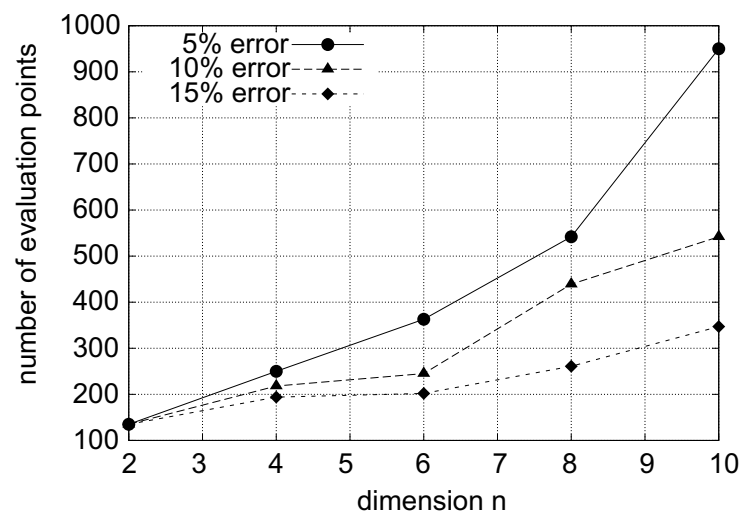

Fig. 10. Number of limit state function evaluations to reach a prescribed precision, as a function of the dimension $n$ of the problem. 
change of the coefficient of variation for the estimates. In this region, relative errors seem to be lower for 20,000 sample points than for 5000 .

From the results summarized in Figs. 7 and 8, it can also be seen that the approximation quality of the MLS method can be enhanced by a small number (between 10 and 20) of additional limit state function evaluations. Thus, most of the limit state functions evaluations are needed to direct the Metropolis algorithm to the region of most probable failure. In comparison with the 50-70 limit state function evaluations necessary in the first study with importance sampling at given MPPs, this smaller number results from the fact that the limit state function evaluations of the optimization procedure for detecting the regions of most probable failure were reused in the MLS algorithm.

The next example has been chosen as to investigate the increase in limit state function evaluations with the number $n$ of random variables involved. For most approximation algorithms, the growth in the number of limit state function evaluations is superlinear. The following limit state function has been studied:

$$
g(\boldsymbol{\theta})=4-\theta_{1}-\sum_{i=2}^{n} \frac{\theta_{i}^{2}}{8},
$$

where $\theta_{i}$ are standard normally distributed random variables. As the Metropolis algorithm seems not to be capable to generate good quality importance sampling densities in higher dimensions, an importance sampling density with unit variance has been introduced at the MPP, which is given by $\theta_{1}=4$ and $\theta_{i}=0, i=2, \ldots, n$. By means of this density, 30,000 sample points were generated for each problem under consideration.

In the following, approximations of the failure probability were computed and further refined by introducing additional evaluation points at which the limit state function is evaluated. From 10 trials, the number of evaluation points such that the approximation error for the failure probabilities falls below a certain limit during three succeeding adaptive refinement steps is recorded and averaged. The results are plotted in Fig. 10 for a $5 \%-, 10 \%$ - and $15 \%$-limit. It can be seen that the growth of the number of limit state function evaluations with dimension $n$ of the problem is dependent on the error level: for an error of $15 \%$ the growth is sublinear, for $10 \%$ almost linear and for $5 \%$, the number of limit state function evaluations grows superlinear with dimension $n$.

\section{Conclusions}

In this paper, a local approximation method for the limit state function in context with reliability computations has been proposed. The method is very versatile, easy to implement and integrates well with existing importance sampling schemes. The method reuses the limit state function evaluations necessary for solving the optimization problem that is connected with the determination of the MPPs. Moreover, the method is capable to interact with the importance sampling scheme in a way that the importance sampling is directed to regions of most probable failures including MPPs that were not detected by the initial optimization algorithm.

Numerical examples have evidenced the fast adaptation of the proposed method and its good approximation quality, even for higher dimensional problems. In this context, it has been noted that often, the error induced by the estimation of the failure probability is larger than the sole approximation error. At least, the latter can be drastically reduced by adding a few base sample points.

The interaction of the proposed method with two importance sampling strategies, based on importance sampling using MPPs and the Metropolis algorithm has been demonstrated in this paper. An integration with other importance sampling techniques seems not to be very difficult and can be done, e.g. by generating arbitrarily the first few evaluation points by means of the sampling density.

\section{References}

[1] Myers RH. Response surface methodology. Boston: Allyn and Bacon; 1971.

[2] Box GEP, Draper NR. Empirical model building and response surfaces. New York: John Wiley; 1987.

[3] Khuri AI, Cornell JA. Response surfaces: designs and analyses. New York: Marcel Dekker; 1996. 
[4] Myers RH, Montgomery DC. Response surface methodology: process and product optimization using designed experiments. New York: John Wiley; 2002.

[5] Box GEP, Wilson KB. The exploration and exploitation of response surfaces: some general considerations and examples. Biometrics 1954;10:16-60.

[6] Faravelli L. Response-surface approach for reliability analysis. J Eng Mech 1989;115(12):2763-81.

[7] Breitung K, Faravelli L. Response surface methods and asymptotic approximations. In: Mathematical models for structural reliability analysis, CRC mathematical modelling series, Boca Raton; 1996. p. 227-85 [chapter 5].

[8] Bucher CG, Bourgund U. A fast and efficient response surface approach for structural reliability problems. Struct Safety 1990;7:57-66.

[9] Rajashekhar MR, Ellingwood BR. A new look at the response surface approach for reliability analysis. Struct Safety 1993;12:205-20.

[10] Kim S-H, Na S-W. Response surface method using vector projected sampling points. Struct Safety 1997;19(1):3-19.

[11] Zheng Y, Das PK. Improved response surface method and its application to stiffened plate reliability analysis. Eng Struct 2000;22:544-51.

[12] Gayton N, Bourinet JM, Lemaire M. CQ2RS: a new statistical approach to the response surface method for reliability analysis. Struct Safety 2003;25(1):99-121.

[13] Wong FA. Slope reliability and response surface method. ASCE J Geotech Eng 1985;111(1):32-53.

[14] Guan XL, Melchers RE. Effect of response surface parameter variation on structural reliability estimates. Struct Safety 2001;23:429-44.

[15] Hurtado JE, Alvarez DA. Neural-network-based reliability analysis: a comparative study. Comput Meth Appl Mech Eng 2001;191:113-32.

[16] Katsuki S, Frangopol DM. Hyperspace division method for structural reliability. J Eng Mech 1994;120:2405-27.

[17] Guan XL, Melchers RE. Multitangent-plane surface method for reliability calculation. J Eng Mech 1997;123(10):996-1002.

[18] Roos D, Bucher CG, Bayer V. Polyhedral response surfaces for structural reliability assessment. In: Proceedings of the eighth international conference on applications of statistics and probability, Sydney, Australia, December 12-15, 1999. Rotterdam: Balkema; 2000. p. 109-15.

[19] Hurtado JE, Alvarez DA, Barbat AH. Monte Carlo analysis of structural systems using neural networks. In: Schuëller GI, Spanos PD, editors. Proceedings of the international conference on Monte Carlo simulation, Monte Carlo, Monaco, June 18-20, 2000. Rotterdam: Balkema; 2000. p. 265-71.

[20] Gomes HM, Awruch AM. Comparison of response surface and neural network with other methods for structural reliability analysis Struct Safety 2004;26(1):49-67.

[21] Schueremans L, Van Gemert D. Benefit of splines and neural networks in simulation based structural reliability analysis. Struct Safety 2005;27(3):246-61.

[22] Deng J. Structural reliability analysis for implicit performance function using radial basis function network. Int J Solids Struct 2006;43:3255-91.

[23] Lancaster P, Salkauskas K. Surface generated by moving least squares methods. Math Comput 1981;37:141-58.

[24] Levin D. The approximating power of moving least squares. Math Comput 1998;67(224):1517-31.

[25] Schuëller GI, Stix R. A critical appraisal of methods to determine failure probabilities. Struct Safety 1987;4:293-309.

[26] Metropolis N, Rosenbluth A, Rosenbluth MN, Teller AH, Teller E. Equation of state calculations by fast computing machines. J Chem Phys 1953;21:1087-92.

[27] Hastings WK. Monte Carlo sampling methods using Markov chains and their applications. Biometrika 1970;57(1):97-109.

[28] Au SK, Beck JL. A new adaptive importance sampling scheme for reliability calculations. Struct Safety 1999;21:135-58.

[29] Bucher CG. Adaptive sampling - an iterative fast Monte Carlo procedure. Struct Safety 1988;5:119-26.

[30] Ang GL, Ang AH-S, Tang WH. Kernel method in importance sampling density estimation. In: Ang AH-S, Shinozuka M, Schuëller GI, editors. Proceedings of fifth international conference on structural safety and reliability, San Francisco, USA; 1989, p. 1193-200. 